\title{
Sum List Coloring Block Graphs
}

\author{
Garth Isaak* \\ Department of Mathematics, Lehigh University, Bethlehem, PA 18015, USA. e-mail: \\ gi02@lehigh.edu
}

\begin{abstract}
A graph is $f$-choosable if for every collection of lists with list sizes specified by $f$ there is a proper coloring using colors from the lists. We characterize $f$-choosable functions for block graphs (graphs in which each block is a clique, including trees and line graphs of trees).

The sum choice number is the minimum over all choosable functions $f$ of the sum of the sizes in $f$. The sum choice number of any graph is at most the number of vertices plus the number of edges. We show that this bound is tight for block graphs.
\end{abstract}

Key words. list coloring, sum coloring, block graphs

\section{Introduction}

List coloring has been well studied in recent years. See for example surveys in [2], $[6],[8],[10]$ or see texts such as [9]. The vertices of a graph (often a line graph) are given color lists and we seek to determine if there is a proper coloring using colors from the lists. Typically one seeks the minimum value $k$ such that for every choice of lists of size $k$ there is a proper coloring. In this case we call the graph $k$-choosable. More generally, a function $f$ specifying the list size for each vertex can be given. The graph is $f$-choosable if for every choice of lists with sizes given by $f$ there is a proper coloring. For block graphs we give necessary and sufficient conditions for a graph to be $f$-choosable. We also describe an inductive test that allows for an efficient algorithm to test $f$-choosability on block graphs.

The ordinary chromatic number of a graph arises from $k$-choosability if all lists are restricted to be initial, of the form $\{1,2, \ldots, k\}$ for some $k$. The (ordinary) chromatic sum of a graph is the minimum sum of colors needed in a proper coloring. We consider the sum choice number of a graph. This is the minimum

* Final version, Feb 2004, to appear in Graphs and Combinatorics 
over all choosable functions $f$ of the sum of the list sizes given by $f$. The chromatic sum arises from the sum choice number if all lists are restricted to be initial.

Simple greedy coloring shows that the sum choice number of any graph is at most the number of vertices plus the number of edges. Our characterization of choosable $f$ for block graphs allows us to show that this bound is tight for these graphs. This includes as special cases trees and line graphs of trees.

In another paper [7] we show that the sum choice number for the cartesian product of $K_{2}$ with $K_{n}$ (the line graph of a complete bipartite graph with one part of size 2$)$ is $n^{2}+\lceil 5 n / 3\rceil$.

We will consider the characterization problem for the special case that each block of the graph is a clique (this contains trees and line graphs of trees). Results on this family of graphs characterizing choosability in terms of given lists with a condition like Hall's Theorem are given in [4]. See also [3] and [5] for more references to related work.

Consider graphs $G$ with vertex set $V(G)$ and edge set $E(G)$ and a collection $\mathcal{C}$ of lists $C(v)$ for $v \in V(G)$. We say that $G$ is $\mathcal{C}$-colorable if there exists a proper coloring with $c_{v} \in C(v)$ such that $v w \in E(G) \Rightarrow c_{v} \neq c_{w}$. Consider a size function $f: V(G) \rightarrow \mathbf{Z}$. We will say that $G$ is $f$-choosable if for every $\mathcal{C}$ with $|C(v)|=f(v)$ for $v \in V$, we have that $G$ is $\mathcal{C}$-colorable. For convenience we allow $f(v) \leq 0$. If any $f(v) \leq 0$ then we will say that $G$ is not $f$-choosable.

The general problem would be to characterize $f$ such that $G$ is $f$-choosable. The list chromatic number (or choice number) of $G$ is the smallest $k$ such that $G$ is $f$-choosable with the constant function $f(v)=k$ for all $v$. We will call $\sum_{v \in V} f(v)$ the size of $f$. The sum choice number $\chi_{S C}(G)$ is the minimum size of a choosable $f$.

We will refer to greedy coloring with respect to a given ordering of the vertices as the coloring obtained by sequentially coloring the vertices in the given order, choosing the smallest color at each step that will not violate the conditions for a proper list coloring. Observe that greedy coloring for given lists may fail to produce a proper coloring.

We will use the elementary fact that greedy coloring does yield a proper coloring for all lists if the list size for each vertex is strictly greater than the number its neighbors that precede it in the greedy ordering. We will say that a list is initial if it is of the form $\{1,2, \ldots, k\}$.

¿From greedy coloring we get the following elementary results, which we record as lemmas for reference later.

Lemma 1 For any graph $G$

$$
\chi_{S C}(G) \leq|V(G)|+|E(G)| .
$$

Proof: Take any ordering of the vertices $v_{1}, v_{2}, \ldots, v_{n}$ and let $f\left(v_{i}\right)$ be one more than the number of vertices in $\left\{v_{1}, v_{2}, \ldots, v_{i-1}\right\}$ adjacent to $v_{i}$. Greedy coloring with this ordering and these list sizes will produce a proper coloring for any lists. 
Lemma 2 Consider a list size function $f$ on the vertices of $K_{n}$. If we order the list sizes from $f$ as $t_{1} \leq t_{2} \leq \cdots \leq t_{n}$ then $K_{n}$ is $f$-choosable if and only if $t_{i} \geq i$ for each $i$.

Proof: If the condition is satisfied, greedy coloring using the ordering given by non-decreasing list sizes yields a coloring. If the condition is not satisfied, say $t_{i}<i$ then using initial lists, fewer than $i$ colors are available to be used on the $i$ vertices corresponding to $t_{1}, t_{2}, \ldots, t_{i}$. So there is no coloring.

A block graph is a graph for which each block is a clique. Let $b(v)$ denote the number of blocks containing vertex $v$. An end block is a block containing one cut vertex. For block graphs we have that $G$ is $f$-choosable if and only if we can allocate $f(v)+b(v)-1$ from each vertex to the cliques so that the condition of Lemma 2 for complete graphs holds on each clique.

\section{Results}

In this section we will state basic results about sum list coloring block graphs. All are closely related and follow from induction using basic lemmas about forced colors and greedy coloring. Proofs will be given in the next section.

Let $B$ be an end block of a graph $G$ with cut vertex $v$ such that $B-\{v\}$ is a component of $G-\{v\}$. Arrange the list sizes for vertices of $B$ other than $v$ in nondecreasing order as $t_{1} \leq t_{2} \leq \cdots$. Let $f \operatorname{force}(f, B)$ be the largest subscript $i$ such that $t_{i} \leq i$, if there is no such $i$ let $\operatorname{force}(f, B)=0$. The idea is that force $(f, B)$ gives the number of colors that can be forced on $B-\{v\}$ by an appropriate choice of lists. With such lists these colors could not be used on $v$.

Lemma 3 A simple inductive procedure to test if $G$ is $f$-choosable: Let $G$ be a graph for which each block is a clique and assume vertex list sizes $f$ are given. Let $v$ be a cut vertex for which all except possibly one of the blocks containing it are end blocks. Let $B_{1}$ be the non-end block (if there are none let it be any block) and let $B_{2}, B_{3}, \ldots B_{b(v)}$ be the other blocks containing $v$. Arrange the list sizes for vertices of $B_{j}$ other than $v$ in nondecreasing order as $t_{j, 1} \leq t_{j, 2} \leq \cdots$.

We have

(i) If $t_{j, i}<i$ for some $j \in\{2,3, \ldots, b(v)\}$ and for some $i$ : Then $G$ is not $f$-choosable.

(ii) If $t_{j, i} \geq i$ for all $j \in\{2,3, \ldots, b(v)\}$ and $i$ : Then let $G^{\prime}$ be the graph obtained by deleting vertices of $B_{2}, B_{3}, \ldots, B_{b(v)}$ other than $v$. Define $f^{\prime}$ on $G^{\prime}$

by restricting $f$ to $G^{\prime}$ except that $f^{\prime}(v)=f(v)-\sum_{j=2}^{b(v)}$ force $\left(f, B_{j}\right)$. Then $G$ is $f$-choosable if and only if $G^{\prime}$ is $f^{\prime}$-choosable.

Let $B_{j}$ be a block of a graph $G$ and recall that $b(w)$ is the number of blocks containing vertex $w$. Sizes $f_{j}(w)$ are called allocated sizes for block $B_{j}$ if $f(w)+$ $b(w)-1=\sum f_{j}(w)$ where the sum is over the blocks containing $w$. Note that non cut vertices are in exactly one maximal clique. So if $w$ is a non cut vertex and $w \in B_{j}$ then $f(w)=f_{j}(w)$. That is, the only non-trivial allocation occurs on cut vertices $u$ where we allocate $f(u)+b(u)-1$ to the cliques containing $u$. 
The idea for the next proposition is that for each cut vertex $w$ we initially increase the list size by $b(w)-1$. Then we attempt to allocate the new sizes for each cut vertex to the blocks containing it in such a way that each block is choosable as in Lemma 2 . The original graph is $f$-choosable if and only if we can make such an allocation.

Proposition 4 A test for $f$-choosability: Let $G$ be a graph for which each block is a clique and assume vertex list sizes $f$ are given. $G$ is $f$-choosable if and only if there exist allocated sizes $f_{j}$ for the maximal cliques such that for each maximal clique $B_{j}$ when the allocated sizes are listed in nondecreasing order as $t_{j, 1} \leq t_{j, 2} \leq \cdots$ we have $t_{j, i} \geq i$ for all $j$ and $i$.

Using the choosability tests described above we can determine the sum choice number for block graphs.

Theorem 5 A formula for the sum choice number of block graphs: Let $G$ be a block graph. Then

$$
\chi_{S C}(G)=|V(G)|+|E(G)| .
$$

Note that trees $T$ are block graphs so this gives $\chi_{S C}(T)=|V(T)|+|E(T)|$. This was first observed by Mike Albertson [1] and can be proved directly by a simple induction.

For sum list coloring edges rather than vertices we apply our results to line graphs $L(G)$. Line graphs of trees are block graphs. It is easy to check that $\mid(V(L(T))|+| E(L(T)) \mid$ can be described in terms of degrees to get the following.

Corollary 6 A formula for the sum edge choice number of trees: Let $T$ be a tree with vertex degrees $d_{1}, d_{2}, \ldots, d_{n}$ and let $L(T)$ be its line graph. Then

$$
\chi_{S C}(L(T))=\sum \frac{d_{i}^{2}}{2} .
$$

For sum list edge coloring trees the allocation from Proposition 4 is as follows. For each non-leaf edge (which corresponds to a cut vertex in the line graph) add one to the size specified by $f$ and allocate this to the ends of the edge in such a way that this list of sizes (in non-decreasing order) for edges incident at each vertex is at least as large term by term as $1,2,3, \ldots, d_{i}$ (where $d_{i}$ is the vertex degree).

\section{Proofs}

The following two basic lemmas are key to the induction for the proofs of the other results. They are versions of elementary results about greedy coloring and degrees in a form that will be useful for our proofs. Both Lemma 7 and Lemma 8 apply to arbitrary graphs.

For a graph $G$ and a set of vertices $S$ we will refer to the open neighborhood $N(S)$ as the set of vertices outside of $S$ which are adjacent to some vertex in $S$. We will say that all vertices in $S$ have the same neighborhood $N(S)$ outside $S$ if $x \in S$ and $y \in N(S)$ implies $x y \in E(G)$. 
Lemma 7 Assume vertex list sizes $f$ are given for $G$ and for some $k$ clique $B$ in $G$ we have $f(v) \leq k$ for every $v \in B$. Assume also that all vertices in $B$ have the same neighborhood $N(B)$ outside $B$. List the sizes assigned to vertices of $B$ in nondecreasing order $t_{1} \leq t_{2} \leq \cdots \leq t_{k} \leq k$. We have

(i) If $t_{i}<i$ for some $i \in\{1,2, \ldots, k\}$ : Then $G$ is not $f$-choosable

(ii) If $t_{i} \geq i$ for all $i \in\{1,2, \ldots, k\}$ : Then let $G^{\prime}$ be the graph induced by $V(G)-B$ and assign list sizes to $G^{\prime}$ as follows: $f^{\prime}(w)=f(w)$ for $w \notin N(B)$ and $f^{\prime}(w)=f(w)-k$ for $w \in N(B)$. Then $G$ is $f$-choosable if and only if $G^{\prime}$ is $f^{\prime}$-choosable.

Proof:

(i): Label the vertices of $B$ as $v_{1}, v_{2}, \ldots, v_{k}$ so that $f\left(v_{i}\right)=t_{i}$. Assigning initial lists to $v_{1}, v_{2}, \ldots, v_{i}$ allows fewer than $i$ colors for this $i$ clique so there is no proper coloring in this case and $G$ is not $f$-choosable.

(ii): Assume that $G$ is $f$-choosable. Given lists $\mathcal{C}^{\prime}$ with sizes $f^{\prime}(v)$ for $v \in$ $V\left(G^{\prime}\right)$, relabel so that the colors are all distinct from $\{1,2, \ldots, k\}$. Form lists $\mathcal{C}$ with sizes $f(v)$ for $v \in V(G)$ by taking $C(v)$ initial of size $f(v)$ for $v \in B$, $C(w)=C^{\prime}(w)$ for $w \in V\left(G^{\prime}\right)-N(B)$ and $C(u)=C^{\prime}(u) \cup\{1,2, \ldots, k\}$ for $u \in N(B)$. Since $G$ is $f$-choosable, there is a proper $\mathcal{C}$-coloring. In any such coloring, colors $1,2, \ldots, k$ must be used on $B$ (since $B$ is a clique and since $t_{i} \geq i$ for all $i$ along with $t_{k} \leq k$ imply $t_{k}=k$ ) and thus not used on $N(B)$. So the coloring restricted to $G^{\prime}$ (after relabelling back to the original colors) is a proper $\mathcal{C}^{\prime}$-coloring.

Conversely, assume that $G^{\prime}$ is $f^{\prime}$-choosable. Given lists $\mathcal{C}$ with sizes $f(v)$ for $v \in V(G)$ color the vertices of $B$ using these lists. Greedy coloring using the ordering given by non-decreasing list sizes in $B$ will yield a coloring because of the condition $t_{i} \geq i$. Let $A$ be the set of colors used on $B$. For $v \in V\left(G^{\prime}\right)$ form lists $\mathcal{C}^{\prime}$ with $C^{\prime}(v)=C(v)-A$. Then the sizes of the $\mathcal{C}^{\prime}$ lists are at least those given by $f^{\prime}$ and hence the remaining vertices (those of $G^{\prime}$ ) are $\mathcal{C}^{\prime}$ colorable. Since the colors in $C^{\prime}$ are distinct from $A$, the coloring of $G^{\prime}$ along with that of $B$ gives a proper $\mathcal{C}$-coloring.

Lemma 8 Assume vertex list sizes $f$ are given for $G$. Let $W$ be a subset of $V(G)$ such that all vertices have the same neighborhood $N(W)$ outside $W$ and let $G^{\prime}$ be the graph induced by $V(G)-W$ and $G^{\prime \prime}$ the graph induced by $W$. Define $f^{\prime}$ on $G^{\prime}$ to be $f$ restricted to $V(G)-W$ and define $f^{\prime \prime}$ on $W$ by $f^{\prime \prime}(w)=f(w)-|N(W)|$ for all $w \in W$. If $G^{\prime \prime}$ is $f^{\prime \prime}$-choosable then $G$ is $f$-choosable if and only if $G^{\prime}$ is $f^{\prime}$-choosable.

Proof: Since $G^{\prime}$ is an induced subgraph of $G$ and on $G^{\prime}$ the list sizes $f^{\prime}$ are the same as $f$, any proper coloring of $G$ using lists with sizes $f$ gives a coloring of $G^{\prime}$ with lists with sizes $f^{\prime}$. So if $G$ is $f$-choosable then $G^{\prime}$ is $f^{\prime}$-choosable.

Conversely, assume that $G^{\prime}$ is $f^{\prime}$-choosable. Given lists $\mathcal{C}$ with sizes $f(v)$ for $v \in V(G)$ color the vertices of $G^{\prime}$ using these lists. Let $A$ be the set of colors used on $N(W)$ in the coloring of $G^{\prime}$. For $w \in W$ form lists $\mathcal{C}^{\prime \prime}$ with $C^{\prime \prime}(w)=C(w)-A$. Then the sizes of the $\mathcal{C}^{\prime \prime}$ lists are at least those given by $f^{\prime \prime}$ and hence the remaining vertices (those of $W$ ) are colorable. Since the colors in $\mathcal{C}^{\prime \prime}$ are distinct 
from $A$, the coloring of $W$ along with that of $V\left(G^{\prime}\right)$ gives a proper $\mathcal{C}$-coloring.

Now the proofs of the propositions follow from applications of the lemmas.

Proof of Lemma 3: This follows from repeated application of Lemmas 7 and 8. Consider first $B_{2}$ and assume vertices of $B_{2}-\{v\}$ are labelled so that $f\left(v_{i}\right)=$ $t_{2, i}$. If $t_{2, i}<i$ for some $i$ then Lemma $7(i)$ applied to $\left\{v_{1}, v_{2}, \ldots, v_{i}\right\}$ shows that $G$ is not $f$-choosable. Otherwise, let $i$ be the largest subscript such that $t_{2, i}=i$ (and if there are none let $i=0$ ). Apply Lemma 8 to $W=v_{i+1}, v_{i+2}, \ldots$, noting that these vertices have a common neighborhood $N(W)=\left\{v, v_{1}, v_{2}, \ldots, v_{i}\right\}$ with size $i+1$. Our assumption gives $f\left(v_{i+l}\right) \geq i+l+1$, so $f^{\prime \prime}\left(v_{i+l}\right)=f\left(v_{i+l}\right)-(i+1) \geq$ $(i+l+1)-(i+1)=l$ and by Lemma $2, W$ is $f^{\prime \prime}$-choosable. So it is enough to consider the case where $B_{2}-\{v\}$ has size $i$ and $t_{2, i}=i$. In this case, force $\left(f, B_{2}\right)=i$. Apply Lemma 7 with $B=B_{2}-\{v\}$, then it is enough to consider the graph $G$ with $B_{2}-\{v\}$ deleted and with size function unchanged except that for $v$ it is equal to $f(v)-f o r c e\left(f, B_{2}\right)$. Repeating the arguments above for $B_{3}, B_{4}, \ldots, B_{b(v)}$ yields the result.

Proof of Proposition 4: We can consider components separately so we may assume that $G$ is connected. Use induction on the number of blocks. The base case for induction is the case that $G$ is a clique and allocation is trivial with $f_{1}(v)=f(v)$ for all $v$ as each vertex is in the only block $B_{1}$. Then Lemma 2 covers this case.

Let $v, B_{1}, B_{2}, \ldots, B_{b(v)}$ be as in Lemma 3 . If for some $j \in\{2,3, \ldots, b(v)\}$ and $i$ when we order the sizes $f$ for vertices of $B_{j}$ other than $v$ we have $t_{j, i}<i$ then by part (i) of Lemma 3 we have that $G$ is not $f$-choosable. But in this case there are at least $i$ vertices $w$ other than $v$ in $B_{j}$ with $f(w)<i$. These are non cut vertices so their allocated sizes are $f_{j}(w)=f(w)$ and no matter how we allocate $f_{j}(v)$, when we order the allocated sizes $f_{j}$ for vertices of $B_{j}$ (including $v$ ) we will have $t_{j, i}<i$.

So we may assume that when we order the sizes $f$ for vertices of $B_{j}$ other than $v$ we have $t_{j, i} \geq i$ for $j \in\{2,3, \ldots, b(v)\}$. Then, by Lemma 3 (ii), $G$ is $f$-choosable if and only if $G^{\prime}$ is $f^{\prime}$-choosable where for vertices $w$ other than $v$ we have $f^{\prime}(w)=f(w)$ and $f^{\prime}(v)=f(v)-\sum_{j=2}^{b(v)} f \operatorname{orce}\left(f, B_{j}\right)$. So by induction it is enough to show that there are allocated sizes $f_{j}^{\prime}$ in $G^{\prime}$ for the maximal cliques such that for each maximal clique $B_{j}$ when the allocated sizes are listed in nondecreasing order as $t_{j, 1}^{\prime} \leq t_{j, 2}^{\prime} \leq \cdots$ we have $i \leq t_{j, i}^{\prime}$ for all $i$ if and only if there are allocated sizes $f_{j}$ in $G$ for the maximal cliques such that for each maximal clique when the allocated sizes for each maximal clique $B_{j}$ are listed in nondecreasing order as $t_{j, 1} \leq t_{j, 2} \leq \cdots$ we have $i \leq t_{j, i}$ for all $i$.

If $G$ has allocated sizes $f_{j}$ satisfying the conditions then for $j \geq 2$, from the definition of $\operatorname{force}\left(f, B_{j}\right)$ we must have $f_{j}(v)>\operatorname{force}\left(f, B_{j}\right)$. So $f(v)+$ $b(v)-1=f_{1}(v)+\sum_{j=2}^{b(v)} f_{j}(v) \geq f_{1}(v)+\sum_{j=2}^{b(v)}\left(\operatorname{force}\left(f, B_{j}\right)+1\right)$ and so $f_{1}(v) \leq$ $\left(f(v)-\sum_{j=2}^{b(v)} \operatorname{force}\left(f, B_{j}\right)\right)=f^{\prime}(v)$. In $G^{\prime}, v$ is in only one block so we must 
allocate $f_{1}^{\prime}(v)=f^{\prime}(v) \geq f_{1}(v)$. For vertices $w$ in $G^{\prime}$ other than $v$ we have $f^{\prime}(w)=$ $f(w)$ and that the number of blocks containing $w$ is the same in $G$ and $G^{\prime}$. For these vertices let $f_{j}^{\prime}(w)=f_{j}(w)$. Then the new allocations satisfy the conditions in $G^{\prime}$ since they satisfied it in $G$ and they are identical except for $f_{1}^{\prime}(v) \geq f_{1}(v)$.

Now assume $G^{\prime}$ has allocated sizes $f_{j}^{\prime}$ satisfying the conditions. Since $v$ is only in one block $B_{1}$ of $G^{\prime}, f_{1}^{\prime}(v)=f^{\prime}(v)=f(v)-\sum_{j=2}^{b(v)} f \operatorname{orce}\left(f, B_{j}\right)$. For vertices $w$ in $G^{\prime}$ other than $v$ let $f_{j}(w)=f_{j}^{\prime}(w)$. (We can do this since $f^{\prime}(w)=f(w)$ for these vertices and they are in the same number of blocks in $G$ and $G^{\prime}$.) In $G$ allocate $f_{1}(v)=f_{1}^{\prime}(v)$ and for $j=2,3, \ldots, b(v)$ allocate $f_{j}(v)=f o r c e\left(f, B_{j}\right)+1$. Then $\sum f_{j}(v)=\left(f(v)-\sum_{j=2}^{b(v)} \operatorname{force}\left(f, B_{j}\right)\right)+\sum_{j=2}^{b(v)}\left(\operatorname{force}\left(f, B_{j}\right)+1\right)=f(v)+$ $b(v)-1$. Vertices $u$ other than $v$ in $B_{2}, B_{3}, \ldots B_{b(v)}$ are in only one block (by the choice of $v$ ) and we must allocate $f_{j}(u)=f(u)$ (where $B_{j}$ is the block containing $u)$.

Recall that we have assumed that when we order the sizes $f$ for vertices of $B_{j}$ other than $v$ we have $t_{j, i} \geq i$ and in addition we have $t_{j, i}>i$ for $i>\operatorname{force}\left(f, B_{j}\right)$ by the definition of $\operatorname{force}\left(f, B_{j}\right)$. Thus in $B_{j}$ with $f_{j}(u)=f(u)$ and $f_{j}(v)=\operatorname{force}\left(f, B_{j}\right)+1$ when we order the vertices of $B_{j}$ (including $v$ ) as $t_{j, 1} \leq t_{j, 2} \cdots$ (note that for $i>\operatorname{force}\left(f, B_{j}\right)$ these $t^{\prime} s$ are shifted by one from those of the first sentence) we have $t_{j, i} \geq i$. For blocks of $G$ other than $B_{2}, B_{3}, \ldots B_{b(v)}$ the allocated sizes are the same as in $G^{\prime}$ and so the condition holds in $G$ also.

We can now prove Theorem 5 .

Proof of Theorem 5: Consider a choosable $f$. By Proposition 4 we can allocate $f(v)+b(v)-1$ so that on each clique $B_{j}$ the allocated values listed in nondecreasing order as $t_{j, 1} \leq t_{j, 2} \leq \cdots$ satisfy $i \leq t_{j, i}$. Hence $\sum(f(v)+b(v)-1) \geq$ $\sum\left(1+2+\cdots+\left|B_{j}\right|\right)$ where the first sum is over vertices and the second is over blocks. This gives $\sum f(v) \geq \sum\left|B_{i}\right|\left(\left|B_{i}\right|+1\right) / 2-\sum(b(v)-1)=|V(G)|+|E(G)|$ as a lower bound. The last equality can easily be checked. The upper bound follows from Lemma 1.

We have shown that $\chi_{S C}(G)=|V(G)|+|E(G)|$ for block graphs. It would be interesting to determine if there are other classes for which this holds.

Acknowledgements. Partially supported by a grant from the Reidler Foundation.

The author would like to thank an anonymous referee for useful comments.

\section{References}

1. Albertson, M.: personal communication, 2001.

2. Alon, N.:, Restricted colorings of graphs. In: K. Walker: Surveys in Combinatorics (London Math. Soc. Lecture Notes Ser. 187, pp. 1-33) Cambridge Univ. Press: 1992. 
3. Cropper, M.M., Goldwasser, J.L., Hilton, A.J.W., Hoffman, D.C., Johnson Jr, P.D.: Extending the disjoint representative theorems of Hall, Halmos and Vaughn to list multicolorings of graphs. J. Graph Theory 33, (2000) 199-219.

4. Hilton, A.J.W, Johnson Jr., P.D.: Extending Hall's Theorem. In: Topics in Combinatorics and Graph Theory: Essays in honor of Gerhard Ringel (pp. 360-371) PhysicaVerlag, Heidelberg: 1990.

5. Hilton, A.J.W., Johnson Jr., P.D.: The Hall number, the Hall index and the total Hall number of a graph. Discrete Appl. Math. 94, (1999) 227-245.

6. Hilton, A.J.W., Slivnik, T., Stirling, D.S.G.: Aspects of List Coloring. Discrete Math. 231, (2001) $254-264$.

7. Isaak, G.: Sum List Coloring 2 by n arrays. Electron. J. Combin. 9, (2002) Note 8, $7 \mathrm{pp}$.

8. Kratochvil, J., Tuza, Z., Voigt, M.: New trends in the theory of graph colorings: choosability and list coloring. In: R. Graham et al.: Contemporary trends in discrete mathematics (DIMACS Ser. Discrete Math. Theoret. Comput. Sci. 49, pp. 183-197) Amer. Math. Soc. Providence: 1999.

9. West, D.B.: Introduction to Graph Theory, 2nd ed. Prentice Hall: 2001.

10. Woodall, D.R.: List Coloring of Graphs. In: J.W.P. Hirschfeld: Surveys in Combinatorics (London Math. Soc. Lecture Notes Ser. 288, pp. 269-301) Cambridge Univ. Press: 2001.

Received: February 2, 2004

Final version received: February 2, 2004 